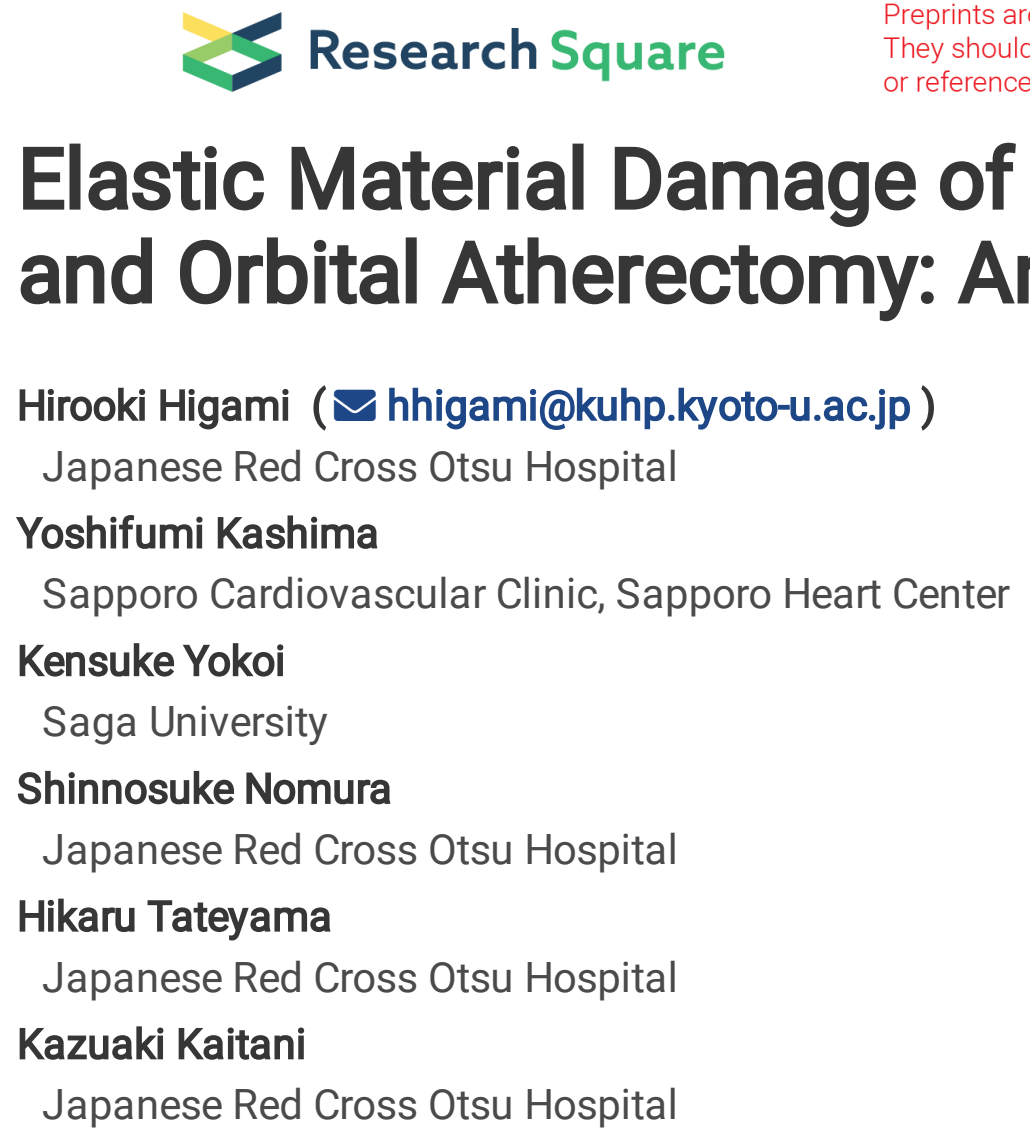

Hirooki Higami ( $\square$ hhigami@kuhp.kyoto-u.ac.jp )

Japanese Red Cross Otsu Hospital

Yoshifumi Kashima

Sapporo Cardiovascular Clinic, Sapporo Heart Center

Kensuke Yokoi

Saga University

Shinnosuke Nomura

Japanese Red Cross Otsu Hospital

Hikaru Tateyama

Japanese Red Cross Otsu Hospital

Kazuaki Kaitani

Japanese Red Cross Otsu Hospital

\title{
Elastic Material Damage of Rotational Atherectomy and Orbital Atherectomy: An in Vitro Assessment
}

\section{Research Article}

Keywords: Rotational atherectomy, orbital atherectomy, disease, rotational speed

Posted Date: January 10th, 2022

DOI: https://doi.org/10.21203/rs.3.rs-1199978/v1

License: (c) (i) This work is licensed under a Creative Commons Attribution 4.0 International License.

Read Full License 


\section{Abstract}

\section{Purpose}

Rotational atherectomy (RA) and orbital atherectomy (OA) are effective procedures for severe calcified coronary artery disease. Nonetheless, vessel perforation remains an adverse complication of these procedures. This study aimed to evaluate factors affecting elastic material damage caused by RA and OA.

\section{Methods}

An in vitro assessment was conducted in which the damage to the rubber latex, an elastic material, after RA was evaluated under various conditions, including burr rotational speed $(100,000-220,000$ rotations per minute), approaching curve, burr size $(1.25 \mathrm{~mm}, 1.75 \mathrm{~mm}$, and $2.0 \mathrm{~mm})$, and fluid viscosity (water and low-molecular weight dextran). Similarly, the rubber latex damage after OA was evaluated in the same experimental system under various conditions, including crown rotational speed, approaching curve, and fluid viscosity.

\section{Results}

In RA, the rubber latex was damaged at lower rotational speeds $(p=0.003)$, tighter approaching curves $(p$ $<0.0001)$, and lower fluid viscosity $(p=0.03)$. In $O A$, the rubber latex was generally severely damaged.

\section{Conclusion}

A higher rotational speed, coaxial approach for the wall, and higher viscosity contributed to lesser elastic material damage in RA. The safety mechanism for elastic material in OA proved less effective.

\section{Introduction}

Percutaneous coronary intervention (PCl) is an effective revascularization technique that is employed in patients with coronary artery disease (CAD). Among those with CAD, severe calcified coronary artery lesions are present in nearly one third of patients undergoing $\mathrm{PCl}$ are at risk for poor clinical outcomes of $\mathrm{PCl}[1-3]$. Thus, calcified lesion modification prior to stent implantation is performed. In this procedure, rotational atherectomy (RA) and/or orbital atherectomy (OA) plays an important role in stent expansion [4-7]. However, they may result in complications, of which vessel perforation is the most serious [8-10]. Thus, a mechanism for preventing vessel perforation in RA should be developed. This safety mechanism has been called the differential cutting (DC) effect. In the DC effect, the rota-burr ablates only calcification and avoids damage for elastic material such as the normal vessel wall. Logically, the safety mechanism for elastic vessel wall is based on the elastohydrodynamic lubrication (EHL) theory [11]; however, the evaluation of the safety mechanism of RA and OA has been limited.

The aim of this study was to evaluate the factors that affected damage for elastic material in RA and OA. 


\section{Methods}

\section{In Vitro Assessment}

To evaluate the factors that affected damage for elastic material under various conditions, we performed in vitro experiments. We molded the simulation models using a 3D printer, which was composed of nylon resin. The system consisted of two parts: the pedestal part and the curve part. The curved parts were molded into three patterns: loose, moderate, and tight curves (Fig. 1). In the role of the virtual elastic vessel wall, rubber latex (thickness $=200 \mu \mathrm{m}$ ), which consisted of sterilized surgical gloves, was rolled along the curved parts (Fig. 2). The guiding catheter (Hyperion, Asahi Intecc, Japan) was equipped with a simulation system and was dipped in the fluid in the form of either water or low-molecular weight dextran. The temperature of the fluid was approximately $20^{\circ} \mathrm{C}$. We evaluated the damage of the elastic material that was rolled along the curved parts in the three degrees of damage during RA or OA. The damage was classified as severe, mild, or no damage with visual recognition. Severe damage corresponded to a complete hole through the material. Mild damage corresponded to some surface damage; however, there was no complete hole. No damage with visual recognition is shown in Fig. 3.

\section{Rotational Atherectomy}

In RA experiments, the wire used was RotaWire Drive Extra Support (Boston Scientific, Massachusetts, USA), and the tip of the wire was pulled using a weight of $15 \mathrm{~g}$. RA was performed using Rotablator Pro (Boston Scientific, Massachusetts, USA). The validated parameters in RA experiments were burr size (1.25 $\mathrm{mm}, 1.75 \mathrm{~mm}$, and $2.0 \mathrm{~mm}$ ), burr rotational speed (100,000 rotations per minute [rpm], 140,000 rpm, $180,000 \mathrm{rpm}$, and 220,000 rpm), and three types of curves (loose, moderate, and tight curves). To evaluate the effect of fluid viscosity on DC, we performed an RA experiment in each burr size and rotational speed condition with a moderate curve system in low-molecular weight dextran instead of water. The rota-burr was manipulated by a well-experienced operator in real-world clinical practice, and the burr was passed through the top of the curve five times or until it reached the maximum ablation time of $20 \mathrm{~s}$. Thus, in the case of burr passage difficulty, the burr either passed the curve below five times or did not at all.

\section{Orbital Atherectomy}

In OA experiments, the wire used was ViperWire Advance Flex Tip (Cardiovascular Systems Inc., St Paul, MN, USA), and the tip of the wire was pulled using a weight of $15 \mathrm{~g}$. OA was performed using Diamondback $360^{\circledR}$ New Classic Crown Type (Cardiovascular Systems Inc., St Paul, MN, USA). The validated parameters in $O A$ experiments were crown rotational speed $(80,000 \mathrm{rpm}$ and $120,000 \mathrm{rpm})$, two types of the curved part (loose and moderate curve), and filled fluid viscosity (water and low-molecular weight dextran). After $\mathrm{OA}$, we evaluated the degree of rubber latex damage using the same criteria as those used in RA experiments.

\section{Statistical Analyses}


In 48 in vitro RA experiments, we investigated factors affecting elastic material damage, which included the burr size, types of curves, burr rotational speed, and filled fluid viscosity, using multivariate analysis of variance. Statistical analysis was conducted by a physician using JMP software version 10.0 (SAS Institute Inc., Cary, NC, USA). Statistical significance was set at $\mathrm{p}<0.05$. The authors had full access to and took full responsibility for the integrity of the data.

\section{Results}

\section{In vitro Rotational Atherectomy Experiments}

In the loose curve system experiments, the rubber latex had no damage, and the burr rotational speed was reduced by approximately $1,000 \mathrm{rpm}$ when the rota-burr passed through the strongest wire-bias point for the wall in all the atherectomy tests. In the moderate curve system experiments, a 1.25-mm burr with a rotational speed of over $180,000 \mathrm{rpm}$ could pass without damage. However, there was mild damage in the 140,000-rpm test and severe damage in the 100,000-rpm test. In the same curve system, a $1.75 \mathrm{~mm}$ burr with a 140,000-rpm rotational speed could pass without damage; however, there was mild damage in the 100,000 -rpm test. In addition, the $2.0 \mathrm{~mm}$ burr could not pass without damage at all rotational speeds. In the tight curve system, the $1.25 \mathrm{~mm}$ burr test caused severe damage to the rubber latex at all rotational speeds. Burrs that were $1.75 \mathrm{~mm}$ and $2.0 \mathrm{~mm}$ in size with over $180,000 \mathrm{rpm}$ rotational speed could pass with mild damage. However, there was severe damage when the two burrs were subjected to the 140,000-rpm test (Table 1). In the comparison of fluid viscosity, $1.25 \mathrm{~mm}$ and $1.75 \mathrm{~mm}$ burrs could pass the moderate curve system without damage in the low-molecular weight dextran in all rotational speed $(100,000 \mathrm{rpm}-220,000 \mathrm{rpm})$. Furthermore, there were some damages in the lower rotational speed with the same burr size and curve in water. Moreover, the $2.0 \mathrm{~mm}$ burr could pass without damage at $220,000 \mathrm{rpm}$ and with mild damage at 140,000 and $180,000 \mathrm{rpm}$ in low molecular dextran. The damage to the rubber latex was lower in the low-molecular weight dextran than in water (Table 2). 
Table 1

Wall damage of rotational atherectomy (experiments in water)

\section{Burr size}

\begin{tabular}{|c|c|c|c|}
\hline & $1.25 \mathrm{~mm}$ & $1.75 \mathrm{~mm}$ & $2.0 \mathrm{~mm}$ \\
\hline Loose curve & \multicolumn{3}{|c|}{ Damage level (rotational speed down [rpm]) } \\
\hline 220000 rpm & $(1000)$ & (1000) & $(1000)$ \\
\hline 180000 rpm & $(1000)$ & (1000) & $(1000)$ \\
\hline 140000 rpm & $(1000)$ & $(1000)$ & $(1000)$ \\
\hline 100000 rpm & (1000) & (1000) & (1000) \\
\hline Moderate curve & \multicolumn{3}{|c|}{ Damage level (rotational speed down [rpm]) } \\
\hline 220000 rpm & (10000) & $(20000)$ & $\triangle(21000)$ \\
\hline 180000 rpm & (10000) & (13000) & $\times(40000)$ \\
\hline 140000 rpm & $\triangle(15000)$ & $(15000)$ & $\times$ (not pass) \\
\hline 100000 rpm & $\times(30000)$ & $\triangle(30000)$ & $\times$ (not pass) \\
\hline Tight curve & \multicolumn{3}{|c|}{ Damage level (rotational speed down [rpm]) } \\
\hline 220000 rpm & $\times(8000)$ & $\triangle(10000)$ & $\triangle(15000)$ \\
\hline 180000 rpm & $\times(10000)$ & $\triangle(10000)$ & $\triangle(20000)$ \\
\hline 140000 rpm & $\times(15000)$ & $\times(20000)$ & $\times(30000)$ \\
\hline 100000 rpm & $\times$ (not pass) & $\times(20000)$ & $\times$ (not pass) \\
\hline
\end{tabular}


Table 2

Wall damage comparison of rotational atherectomy between water and low-molecular weight dextran

Water

Low-molecular weight dextran

Burr size: $1.25 \mathrm{~mm}$

\begin{tabular}{|ccc}
$220000 \mathrm{rpm}$ & $\bullet(10000)$ & $\bigcirc(9000)$ \\
$180000 \mathrm{rpm}$ & $(10000)$ & $(9000)$ \\
$140000 \mathrm{rpm}$ & $\triangle(15000)$ & $(6000)$ \\
$100000 \mathrm{rpm}$ & $\times(30000)$ & $\bigcirc(10000)$
\end{tabular}

Burr size: $1.75 \mathrm{~mm}$

$\begin{array}{lll}220000 \mathrm{rpm} & \bullet(20000) & \bullet(9000) \\ 180000 \mathrm{rpm} & \bullet(13000) & \bullet(10000) \\ 140000 \mathrm{rpm} & \bullet(15000) & \bullet(9000) \\ 100000 \mathrm{rpm} & \triangle(30000) & \bigcirc(10000)\end{array}$

Burr size: $2.00 \mathrm{~mm}$

$\begin{array}{lll}220000 \mathrm{rpm} & \triangle(21000) & \bullet(20000) \\ 180000 \mathrm{rpm} & \times(40000) & \triangle(21000) \\ 140000 \mathrm{rpm} & \times \text { (not pass) } & \triangle(22000) \\ 100000 \mathrm{rpm} & \times \text { (not pass) } & \times \text { (not pass) }\end{array}$

: no damage, $\triangle$ : mild damage (not complete hole but surface damage), $x$ : severe damage (complete hole)

\section{Effective Factors for Differential Cutting in Rotational Atherectomy}

Multivariate analysis was performed to determine the factors that could significantly influence the elastic material damage, including the degree of approaching curve, rotational speed, burr size, and fluid viscosity. The degree of curve was the most significant factor (moderate-loose, $p<0.0001$; tightmoderate, $p=0.013)$. Among the controllable factors, rotational speed was the most significant $(p=$ $0.0032)$; subsequently, fluid viscosity was significant $(p=0.03)$ (Table 3$)$. 
Table 3

Multiple regression analysis for wall damage of rotational atherectomy

\begin{tabular}{|lccc|}
\hline Term & Estimate & Chi-square & P value \\
\hline Curve; moderate-loose & -18.6 & 100000 & $<.0001$ \\
\hline Curve; tight-moderate & -2.51 & 6.16 & 0.013 \\
\hline Higher burr rotational speed (rpm) & 0.00 & 8.7 & 0.0032 \\
\hline Bigger burr size (mm) & -2.03 & 2.46 & 0.12 \\
\hline Low-molecular weight dextran & 1.08 & 4.70 & 0.03 \\
\hline p value for the whole model < 0.0001, R square = 0.49 & & \\
\hline (Wall damage level was defined as per the following ordinal index; severe: 2, mild: 1 , none: 0$)$ \\
\hline
\end{tabular}

\section{In Vitro Orbital Atherectomy Experiments}

The OA test could be performed in $20 \mathrm{~s}$ without rubber latex damage if the rotational speed was 80,000 rpm and if low-molecular weight dextran was used. Other tests under various conditions, including low and high rotational speed and fluid viscosity (water or low-molecular weight dextran), caused severe damage to the latex (Table 4). In the OA experiments, we confirmed that there were multiple holes on the rubber latex, whereas in the RA experiments, those with severe damage to the rubber latex had a single complete hole (Fig. 4).

Table 4

Wall damage assessment of orbital atherectomy

Water Low-molecular weight dextran

Loose curve

80000 rpm

$120000 \mathrm{rpm}$

$\times$

$\times \quad x$

Moderate curve

80000 rpm

$x$

X

$120000 \mathrm{rpm}$

$x$

$x$

: no damage; $\triangle$ : mild damage (not complete hole but surface damage); $x$ : severe damage (complete hole)

\section{Discussion}

The primary findings of this study are as follows: 1 ) in the RA procedure, the factors that affected the safety mechanism to avoid damage to the elastic material included a larger minimum turning radius of 
the approaching curve, higher burr rotational speed, and higher viscosity of the filled fluid; 2) in the OA procedure, the safety mechanism for elastic material was less effective than RA.

The EHL theory was based on the classical one-dimensional Reynolds equation, $P S=6 \eta u / h$. In this equation, $P$ is the pressure between the rotational material and the opposite wall, $\eta$ is the viscosity, $u$ is the speed of the moving surface, and $h$ is the distance between the rotational material and the opposite wall [11]. Currently, several tenets, including elastic deformation caused by fluid pressure, have been included in the theory. According to the EHL theory, laminar fluid flow caused by rotational forces creates pressure between the rotational material and the opposite wall. During RA, this pressure causes microelastic deformation in the elastic vessel wall and maintains the distance between the rota-burr and the vessel wall. This phenomenon seems to be the key mechanism within the safety mechanism of the rotablator; therefore, the present in vitro study has attempted to demonstrate this logic. We found that a higher burr speed and a higher viscosity were effective for preventing elastic damage; however, a larger burr size was not effective. Subsequently, we must pay attention to fluid viscosity and control optimal rotational speed during the RA procedure. The viscosity of $\mathrm{H}_{2} \mathrm{O}$ at $20^{\circ} \mathrm{C}$ is approximately $1.0 \mathrm{mPa} \square \mathrm{s}$, and that of low-molecular weight dextran is 3.5-4.5 Pa\s. In real-world RA procedure, fluid viscosity represents the blood viscosity. Low-molecular weight dextran is reported to be the same as $50 \%$ hematocrit blood viscosity [12]. With respect to the correlation between blood viscosity and hematocrit and between blood viscosity and blood protein levels [13], patients with severe anemia and low blood protein levels may not be expected to have a good DC effect during RA. In such cases, transfusion and/or supplementation of albumin before $\mathrm{PCl}$ may be required.

Logically, a larger burr size results in a higher speed of the moving surface and increases the DC effect. However, the shape of the rota-burr depends on the burr size, and the approaching degree of the vessel wall is more coaxial in the 1.25-mm burr than in the 2.0-mm burr (Online Resource 1). As suggested in a previous study [14], the difference in shape results in a higher perpendicular reaction force of the opposite wall in a 2.0-mm burr than in a 1.25-mm burr. This might have caused a missing DC effect in the larger rota-burr (Online Resource 2). Moreover, the surface point of the rota-burr attached to the vessel wall is not necessary at the maximum radius point; therefore, the theory that a larger burr size increases the safety for elastic material is not necessarily true. For the same reason, a tighter approaching curve results in a higher perpendicular reaction force between the rota-burr and the vessel wall; therefore, a tighter curve may decrease the safety for elastic material. However, a higher perpendicular reaction force teaches procedural operators the power limitation of pushing the burr controller; thus, well-experienced operators might avoid vessel perforation before the perpendicular reaction force becomes excessive. In clinical practice, $\mathrm{PCl}$ operators should evaluate the strong wire bias for the tight curve in the normal vessel wall via angiography and imaging devices such as optical coherence tomography and/or intravascular ultrasound when the rota-burr must pass through the tortuous point in the rota-mode. Regarding the burr rotational speed, the Boston Scientific formally recommended a speed of 140,000-190,000 rpm [15]. With respect to the safety effect for the elastic vessel wall, this speed is adequate; however, excess burr speed 
might result in thrombus formation. Therefore, operators should consider the appropriate settings for each rotational atherectomy case.

Khan et al. reported that the incidence of coronary perforation was significantly higher in OA patients than in RA patients [16]. In the orbital atherectomy system, the center of gravity of the asymmetric crown is far from the wire, which is the axis of rotation; therefore, the rotational surface is not continuous. This makes it difficult to produce a laminar fluid flow and apply the EHL theory. In the RA in vitro experiment, the rubber latex could not be damaged in the loose curve system under various conditions, even at low rotational speeds. However, OA could easily damage the same model without $80,000 \mathrm{rpm}$ in lowmolecular weight dextran. Although a higher viscosity might confer the benefit of possibly avoiding vessel damage in $\mathrm{OA}$, a strong wire bias point for the elastic vessel wall should be mentioned for severe vessel perforation in OA more than RA. Moreover, perforation caused by OA may be more complex than that caused by RA.

This study has several limitations. First, this was an in vitro assessment. To evaluate the damage of the rubber latex that might have been tougher than the real vessel wall, we prepared a strong wire bias by pulling the wire using a weight. Second, the curve models were not duplications of the real coronary artery. Thus, the approaching degree for the wall of the rota-burr or OA catheter might differ from realworld practice. Moreover, in the present study, we investigated the damage on the strong forced inner side of curve. In the RA practice, the perforation was experienced at the outer side of curve just after the tortuous vessel route. This might be related burr manipulation. However, it seemed to be essentially the same principle of the safety mechanism for elastic material in RA. Therefore, the results of the experiment should be interpreted as a comparative assessment in various conditions, including rotational speed of the rota-burr or OA catheter, rota-burr size, fluid viscosity, approaching curve, and RA or OA.

From a clinical perspective, RA has an advantage in its safety mechanism compared to OA. Moreover, RA operators could decrease the possibility of vessel perforation with controlling factors, higher rotational speed, and blood viscosity.

In conclusion, a higher rotational speed, coaxial approach for the wall, and higher viscosity contribute to the safety mechanism of RA. The safety mechanism for elastic material in OA proved less effective.

\section{Declarations}

\section{Acknowledgements}

The authors thank Boston Scientific Corporation and Asahi Intecc for providing us with the sample devices.

\section{Funding}

The authors declare that no funds, grants, or other support were received during the preparation of this manuscript. 


\section{Competing Interests}

The authors have no relevant financial or non-financial interests to disclose.

\section{Author Contributions}

All authors contributed to the study conception and design. Material preparation, data collection, and analysis were performed by $\mathrm{HH}$ and $\mathrm{HT}$. The first draft of the manuscript was written by $\mathrm{HH}$, and all authors commented on previous versions of the manuscript. All authors read and approved the final manuscript.

\section{Ethics Approval}

This study did not use biological data or clinical data. Therefore, there was no ethical approval. The Japanese Red Cross Otsu Hospital Research Ethics Committee has confirmed that no ethical approval is required.

\section{Consent to Participate}

Any human participants was not involved in this study.

\section{References}

1. Madhavan, M. V. et al. Coronary artery calcification: pathogenesis and prognostic implications. J. Am. Coll. Cardiol. 63, 1703-1714 (2014).

2. Ertelt, K. et al. Impact of the severity of coronary artery calcification on clinical events in patients undergoing coronary artery bypass grafting (from the Acute Catheterization and Urgent Intervention Triage Strategy Trial). Am. J. Cardiol. 112, 1730-1737 (2013).

3. Généreux, P. et al. Two-year outcomes after percutaneous coronary intervention of calcified lesions with drug-eluting stents. Int. J. Cardiol. 231, 61-67 (2017).

4. Kobayashi, Y. et al. Impact of target lesion coronary calcification on stent expansion. Circ. J. 78, 2209-2214 (2014).

5. Taniwaki, M. et al. Mechanisms of very late drug-eluting stent thrombosis assessed by optical coherence tomography. Circulation 133, 650-660 (2016).

6. Kang, S. J. et al. Mechanisms of in-stent restenosis after drug-eluting stent implantation: intravascular ultrasound analysis. Circ. Cardiovasc. Interv. 4, 9-14 (2011).

7. Généreux, P. et al. Ischemic outcomes after coronary intervention of calcified vessels in acute coronary syndromes. Pooled analysis from the HORIZONS-AMI (Harmonizing Outcomes with revascularization and Stents in acute myocardial infarction) and ACUITY (Acute Catheterization and Urgent Intervention Triage Strategy) TRIALS. J. Am. Coll. Cardiol. 63, 1845-1854 (2014). 
8. Januszek, R., Siudak, Z., Dziewierz, A., Dudek, D. \& Bartuś, S. Predictors of in-hospital effectiveness and complications of rotational atherectomy (from the ORPKI Polish National Registry 2014-2016). Catheter. Cardiovasc. Interv. 92, E278-E287 (2018).

9. Kinnaird, T. et al. Incidence, determinants, and outcomes of coronary perforation during percutaneous coronary intervention in the United Kingdom between 2006 and 2013: an analysis of 527121 cases from the British Cardiovascular Intervention Society database. Circ. Cardiovasc. Interv. 9, e003449 (2016).

10. Kinnaird, T. et al. Operator volumes and in-hospital outcomes: an analysis of 7,740 rotational atherectomy procedures from the BCIS National database. JACC Cardiovasc. Interv. 14, 1423-1430 (2021).

11. Greenwood, J. A. Elastohydrodynamic lubrication. Lubricants 8, 51 (2020).

12. Yao, S. T. \& Shoemaker, W. C. Plasma and whole blood viscosity changes in shock and after dextran infusion. Ann. Surg. 164, 973-984 (1966).

13. Grotta, J., Ostrow, P., Fraifeld, E., Hartman, D. \& Gary, H. Fibrinogen, blood viscosity, and cerebral ischemia. Stroke 16, 192-198 (1985).

14. Sakakura, K. et al. When a burr cannot penetrate the calcified lesion, increasing burr size as well as decreasing burr size can be a solution in rotational atherectomy. Int. Heart J. 58, 279-282 (2017).

15. Sakakura, K. et al. Incidence and determinants of complications in rotational atherectomy: insights From the National Clinical Data (J-PCI Registry). Circ. Cardiovasc. Interv. 9, e004278 (2016).

16. Khan, A. A. et al. Outcomes of rotational atherectomy versus orbital atherectomy for the treatment of heavily calcified coronary stenosis: a systematic review and meta-analysis. Catheter. Cardiovasc. Interv., 1-9 (2020).

\section{Figures}




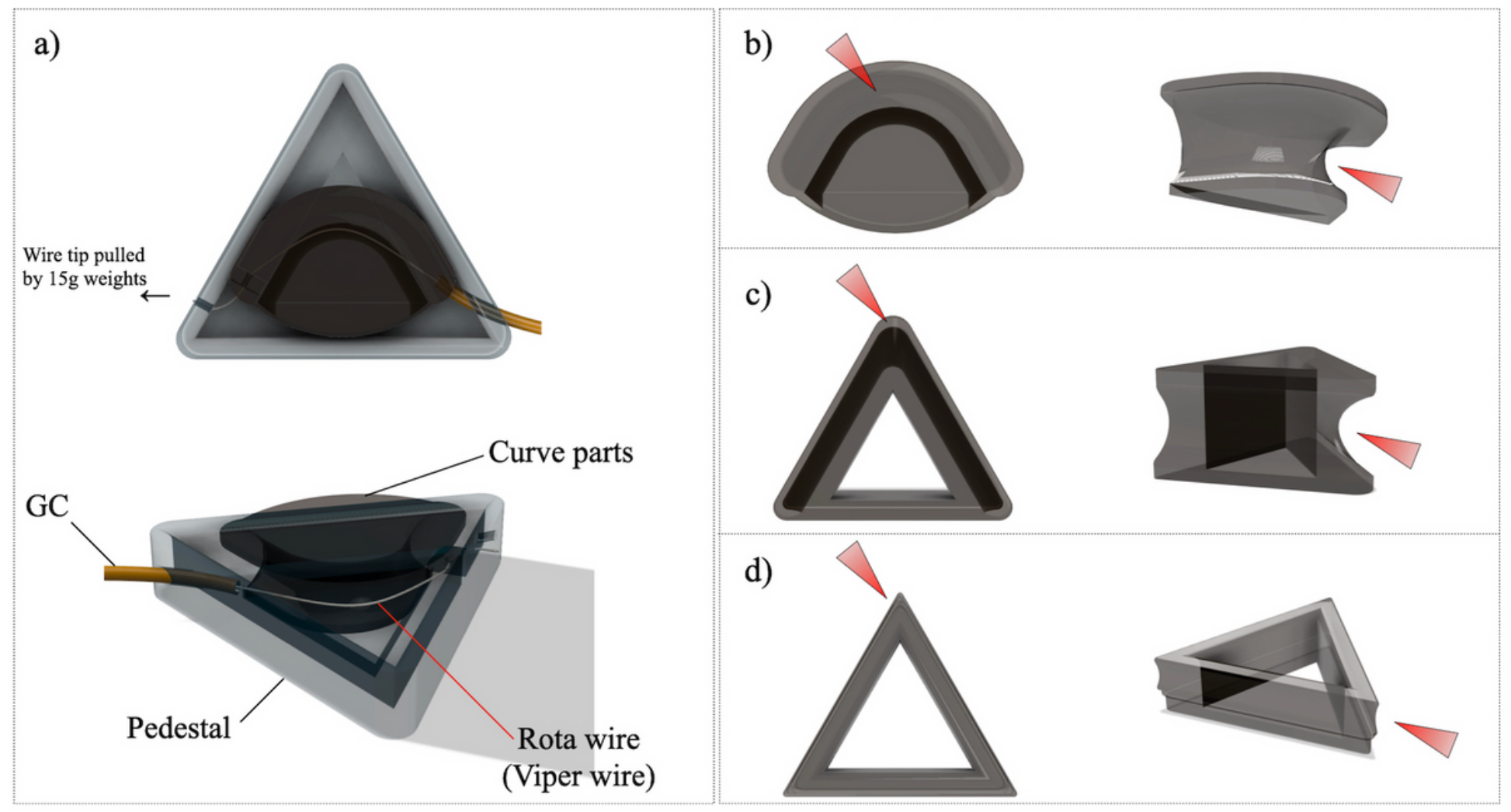

\section{Figure 1}

System parts used in the in vitro experiments. All experimental system parts without a wire, rotablator, orbital atherectomy catheter, and guiding catheter were made using a three-dimensional printer with nylon resin. (a) The pedestal part is set on the bottom of the water tank, and the curved part is engaged on the pedestal part. A straight guiding catheter (GC) is inserted and placed in the side hole of the pedestal part. The tip of the wire is removed from the water tank through the side hole on the tank wall and pulled by a $15 \mathrm{~g}$ weight. (b) Loose curve part. The red arrow shows the groove of the curve passing through the wire. (c) Moderate curve part. The red arrow shows the groove of the curve passing through the wire. (d) Tight curve part. The red arrow shows the groove of the curve passing through the wire. 

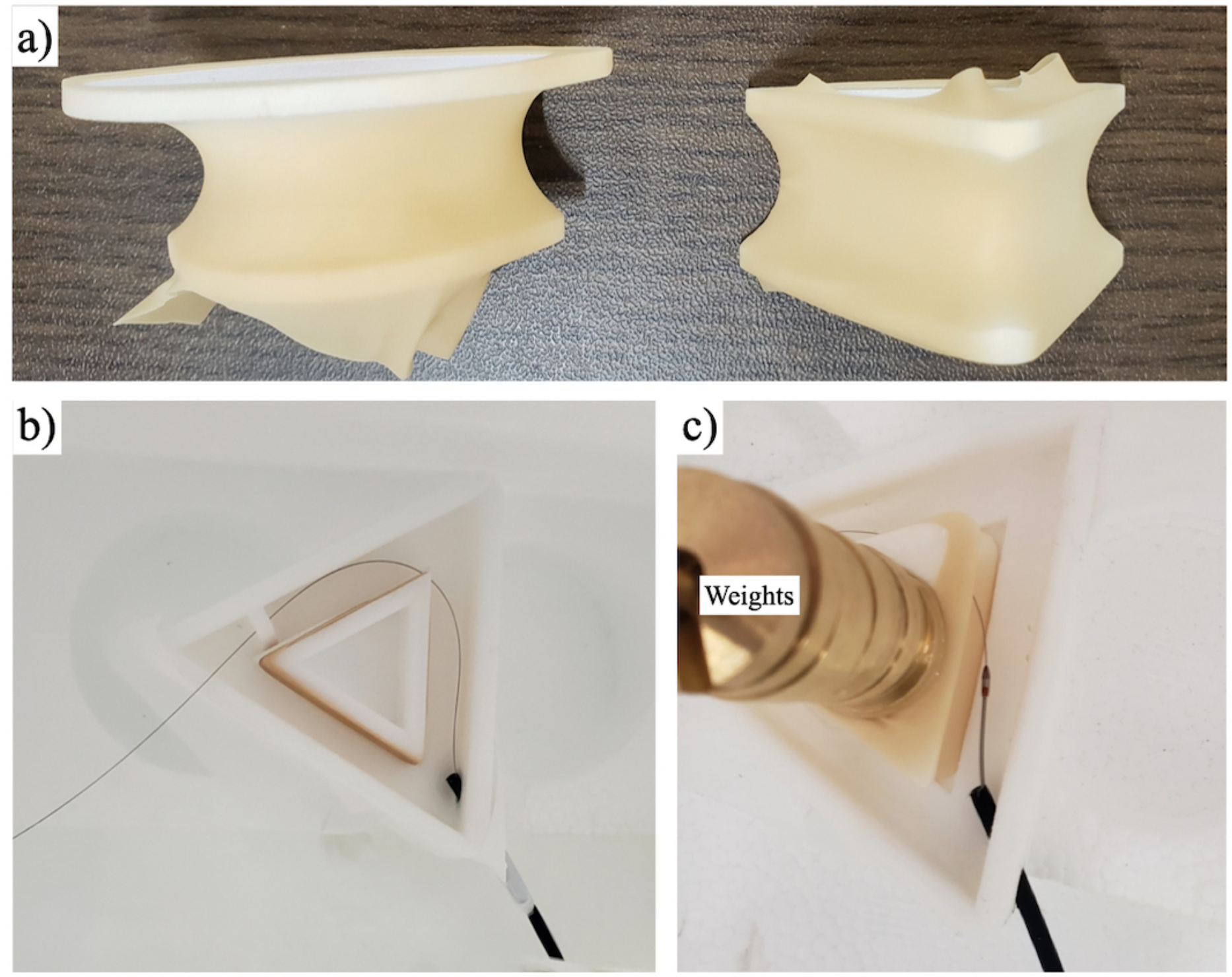

\section{Figure 2}

Rolled-up rubber latex as elastic material for the curve parts. (a) Representative settings of the rolled-up rubber latex around the curve parts (left: loose curve, right: moderate curve). (b) Real representative experiment system (curve part: tight curve, wire: RotaWire Drive Extra Support). (c) Real representative experiment system (curve part: moderate curve, wire: RotaWire Drive Extra Support, and $1.25 \mathrm{~mm}$ rota-burr on the wire). During rotational atherectomy or orbital atherectomy, enough weights pulled on the curve part ensuring system stability. 
a)

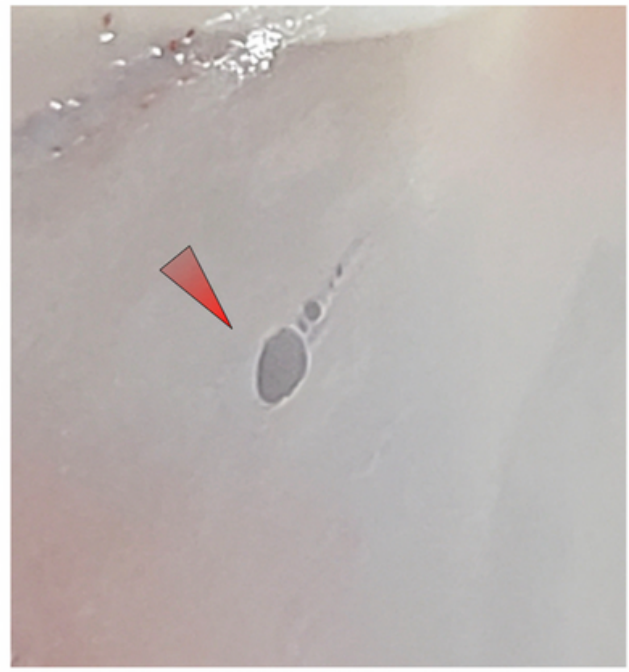

b)

c)

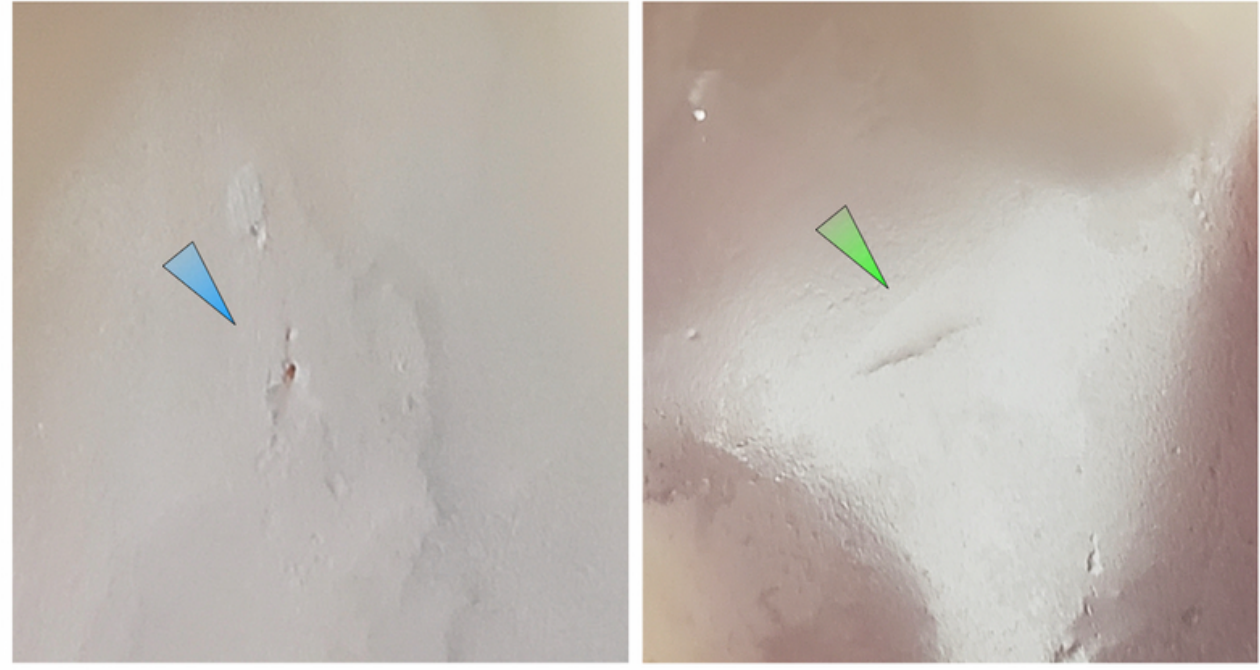

\section{Figure 3}

Criteria for damage on the rubber latex. (a) Severe damage: rubber latex had a complete hole. (b) Mild damage: some visible damage on the surface of the rubber latex without a complete hole. (c) No damage: there was no visible damage on the rubber latex.

a)

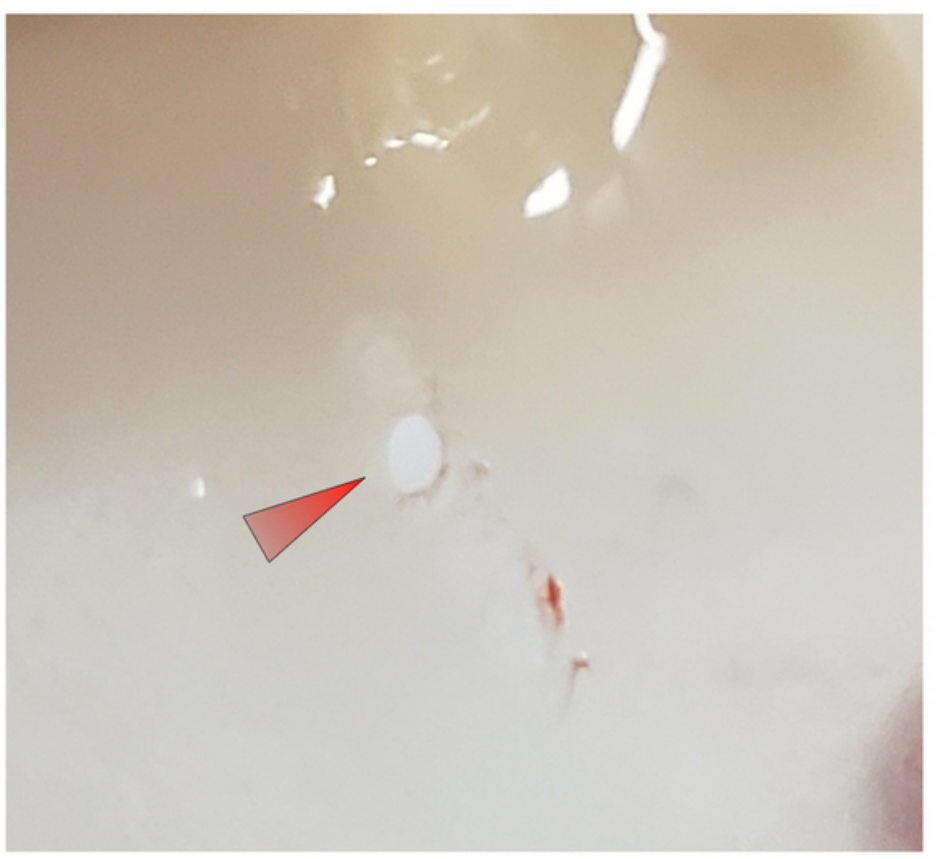

Rota: Moderate curve, $\mathrm{H}_{2} \mathrm{O}, 2.0 \mathrm{~mm}, 180000 \mathrm{rpm}$ b)

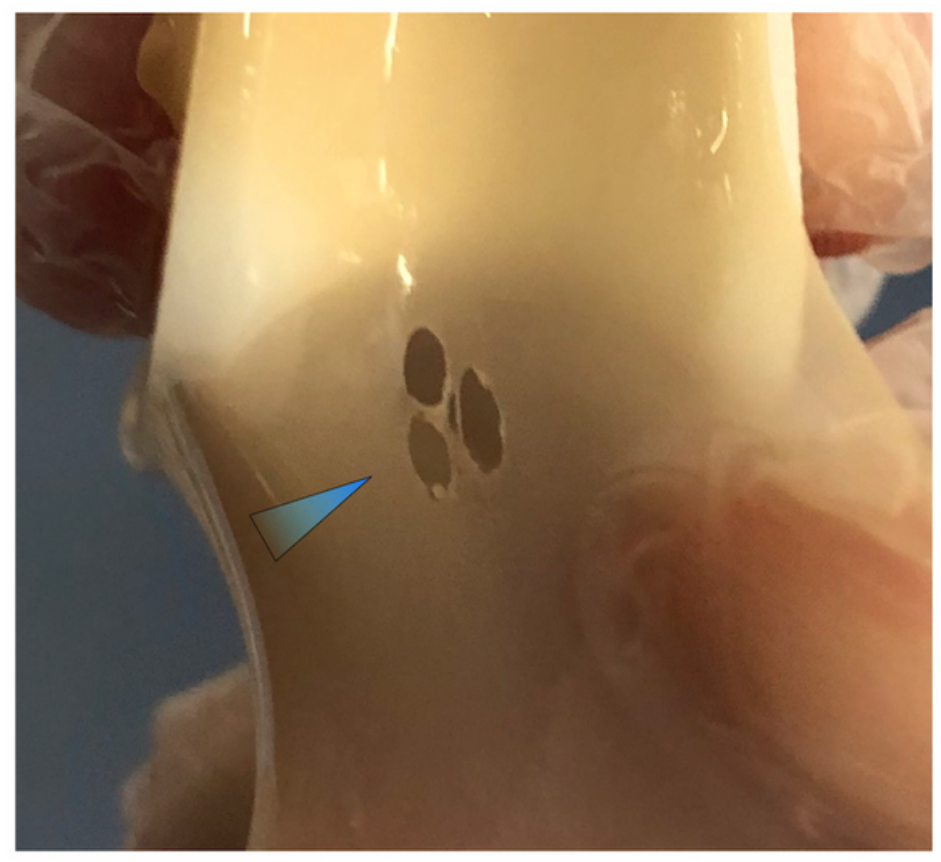

OAS: Moderate curve, $\mathrm{H}_{2} \mathrm{O}, 120000 \mathrm{rpm}$

\section{Figure 4}

Comparison of the shape of the damage on rubber latex between rotational atherectomy and orbital atherectomy. (a) Representative single complete hole after rotational atherectomy (moderate curve 
system, in water, burr size: $2.0 \mathrm{~mm}, 180,000 \mathrm{rpm}$ rotational speed). (b) Representative multiple complete holes after orbital atherectomy (moderate curve system, in water, 120,000 rpm rotational speed). 